\title{
Putting antimicrobial resistance in the corner
}

\author{
Transgenic corn expressing anti-inflammatory antibodies presents a new avenue for mitigation of the enteric \\ disease coccidiosis, providing new possibilities for antimicrobial-free management of poultry diseases.
}

\section{Charles Li and Mingmin Lu}

T he use of antibiotics as growth promoters has been common practice in food animal production for decades. Alongside bacteriostatic and/ or bactericidal activity, antibiotics mediate growth enhancement by tuning the overall density or diversity of the gut microbiota, thus optimizing nutrient utilization, or by reducing energy waste and improving production $^{1}$. Together with food safety, animal welfare and infectious disease control, antimicrobial resistance remains a growing threat to both animals and humans. In this issue of Nature Food, Lessard and colleagues present an alternative approach to antibiotics by feeding chickens with transgenic corn expressing an antibody specific for chicken interleukin-10 (anti-cIL-10), with the aim of mitigating coccidiosis severity ${ }^{2}$.

Coccidiosis - one of the most common enteric infectious diseases - accounts for losses of approximately US $\$ 3$ billion annually to the poultry industry worldwide ${ }^{3}$, negatively impacting poultry welfare and reducing production performance by lowering feed intake and weight gain. One strategy for preventing such losses includes the use of live and slightly attenuated coccidia vaccines. However, potential side effects are intestinal damage in the epithelia and mucogenesis, predisposing chickens for secondary Clostridium perfringens proliferation and increasing the incidence of necrotic enteritis ${ }^{4}$. It is therefore common to use ionophore antibiotics in a prophylactic manner, but this runs a serious risk of antimicrobial resistance and fails to meet consumer demands for 'antibiotic-free' poultry products.

Eimeria - the species of parasite that causes coccidiosis - induces excess local production of intestine luminal IL-10, a potent anti-inflammatory and suppressive cytokine. Excess IL-10 production can enhance coccidiosis pathogenesis by supressing immune responses and facilitating coccidiosis progression ${ }^{5}$. Genetic abolition of IL-10 and IL-10 receptor obstruction resulted in effective clearance of many microbial pathogens, including parasitic, bacterial and viral pathogens ${ }^{6}$. Thus, Lessard and co-authors developed single-domain antibodies (sdAbs) that were able to bind to cIL-10, block cIL-10 receptor binding and resume interferon- $\gamma$ secretion from cIL-10 repressed primary chicken splenocytes ${ }^{2}$. Previously, an anti-cIL-10 antibody prepared using egg yolk powder from chickens immunized with cIL-10 peptides was tested as a feed additive, and improvements in animal growth and gut health of Eimeriachallenged broiler chicks were observed ${ }^{5}$. However, egg-yolk-derived antibodies have several limitations, including high production expenses, poor thermal stability of immunoglobulin Y proteins, and potential pathogenic cross-contamination issues from animal source byproducts. To address these pitfalls, Lessard et al. used microbially produced anti-cIL-10 sdAbs with good thermal stability (retaining $50 \%$ binding affinity of the antibody to cIL- 10 at $95^{\circ} \mathrm{C}$ for $3 \mathrm{~min}$, and $15 \%$ at $95^{\circ} \mathrm{C}$ for $\left.30 \mathrm{~min}\right)^{2}$ and expressed the antibodies in transgenic corn for processing by feed pelleting. This approach addressed three practical and important issues: it reduced the risk of cross-contamination of avian infectious pathogens by avoiding avian products during production; improved the stability of the antibody at high temperatures, allowing feed pellets to be processed using standard feed protocols; and reduced the cost of antibody production by utilizing corn instead of more conventional and expensive antibody production methods.

The feeding trials conducted by Lessard et al. demonstrate the efficacy of transgenic corn expressing anti-cIL-10. Birds were fed transgenic corn (or control non-transgenic corn) for 14 days, after which a mixture of oocysts from the three most common coccidiosis-causing Eimeria species (Eimeria acervulina, Eimeria maxima and Eimeria tenella) were administered by oral gavage (alongside Eimeria-free water controls). Compared to the Eimeria-challenged untreated controls, chickens that were fed transgenic corn - especially those fed the highest dosage of $1.6 \mathrm{~kg}$ grain per $100 \mathrm{~kg}$ standard corn-soy diet - demonstrated improved 20-day body weight gain, normal feed conversion ratios and reduced oocyst counts (except for E. maxima) and lesion scores. Chickens that were fed transgenic corn had comparable weight gain and feed conversion results to Eimeria-challenged non-transgenic corn-fed birds treated with the coccidiostat ionophore antibiotic salinomycin. The protection efficacies observed were good, given that considerably high dosages of three mixed Eimeria species pathogens were administered (50,000100,000 oocysts per animal for each Eimeria species). The transgenic corn approach presents a potentially valuable real-world option for antibiotic-free management of Eimeria infections, while also avoiding complicated and costly antibody formulation and administration steps.

Future efforts should be made to determine whether transgenic corn expressing CIL-10 antibodies also has beneficial effects for controlling other pathogen infections. A prime example is C. perfringens, another common enteric infectious pathogen for the poultry industry that causes necrotic enteritis, especially following withdrawal of antibiotic growth promoters. Availability of such data would aid in the urgent pursuit of antibiotic alternatives. The approach demonstrated here presents new hope in the fight against food-production-related antimicrobial resistance, which benefits not only animal welfare but also human health.

Charles Li ${ }^{\bowtie}$ and Mingmin Lu Beltsville Agricultural Research Center, Agricultural Research Service, US Department of Agriculture, Beltsville, MD, USA.

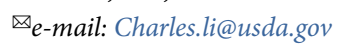

Published online: 10 February 2020 https://doi.org/10.1038/s43016-020-0034-9

References

1. Gadde, U. et al. Anim. Health Res. Rev. 18, 26-45 (2017).

2. Lessard, P. A. et al. Nat. Food https://doi.org/10.1038/s43016-020 0029-6 (2020).

3. Li, R. et al. Vet. Parasitol. 243, 79-84 (2017).

4. Collier, C. T. et al. Vet. Immunol. Immunopathol. 122, 104-115 (2008).

5. Arendt, M. K. et al. Poult. Sci. 95, 430-438 (2016).

6. Gautam, S. et al. J. Infect. Dis. 204, 1134-1137 (2011). 